\title{
Difficulties in Diagnosis of Malaria in Non-Endemic Areas: A Case Report of a Child in Brazil
}

Yvone Maia Brustoloni' ${ }^{*}$, Pamela Mantovani Baldissera ${ }^{1}$, Fernanda Maia Brustoloni $^{2}$ and Maria Elizabeth Cavalheiros Dorval ${ }^{1}$

${ }^{1}$ Federal University of Mato Grosso do Sul, Campo Grande, Mato Grosso do Sul, Brazil

${ }^{2}$ Anhanguera-UNIDERP University, Campo Grande, Mato Grosso do Sul, Brazil

\begin{abstract}
Malaria is a tropical parasitic disease that triggers severe social and economic problems worldwide and causes thousands of deaths each year. Early diagnosis enables proper treatment. People from non-endemic areas who travel to areas of transmission are often exposed to late or incorrect diagnosis because healthcare professionals have great difficulty in conceiving malaria as a diagnostic hypothesis. In addition, technicians skilled in recognizing malaria etiologic agent are not abundant. This paper addresses the case of a child from a non-endemic malaria region in Brazil, with a prior hypothetic diagnosis of visceral leishmaniasis, however bone marrow aspirate revealed Plasmodium instead of Leishmania. A retrospective epidemiological investigation showed a history of a recent trip to a malaria area and the review of blood smears obtained on admission disclosed a large number of trophozoites and gametocytes of Plasmodium falciparum that had been unnoticed, suggesting that diagnosis could have been possible earlier. Malaria clinical picture is easily mistaken for other febrile infectious diseases, thus the possibility of its occurrence cannot be ruled out. There is a need to increasingly train the microscopy technicians, both in endemic and non-endemic areas, so as to minimize the false diagnoses and decrease morbidity and mortality of the disease.
\end{abstract}

Keywords: Malaria; Plasmodium falciparum; Leishmaniasis; Diagnosis

\section{Background}

Although malaria incidence and mortality rates have fallen during the past decade, its transmission still occurs in 99 countries around the world. In 2010 there were an estimated 216 million cases with about 655,000 deaths, mainly among children under five years of age [1] and in Brazilian Amazon there were 305 (37.8\%) municipalities with malaria epidemics [2]. Malaria is still a major public health problem in Brazil, with approximately 300.000 cases registered yearly, almost exclusively $(99,8 \%)$ restricted to the region of the Amazon Basin [3]. We here describe a case of a boy residing in a non-endemic area of malaria in Brazil who had travelled to a state of the Amazon Basin, and whose diagnosis of malaria has not been hypothesized when he presented fever some days after he returned home. Malaria diagnosis was completed by the finding of trophozoites and gametocytes of Plasmodium falciparum in bone marrow aspirate. Parasites were present on blood smears since admission but had gone unnoticed. The case here described illustrates that out of endemic areas general practitioners are much less alert to consider the diagnosis of malaria when facing a febrile patient.The objective of this report is to highlight that malaria must be considered as a potential diagnosis in diseases with intermittent fever. Currently, migration and travels require sentinel surveillance of febrile patients. Lost or late diagnosis of malaria, specially caused by P. falciparum, can lead to severe outcomes.

\section{Case Report}

A boy aged 11 years and eight months, coming from the state of Mato Grosso do Sul, mid-western region of Brazil, was taken to the teaching hospital of the Federal University of Mato Grosso do Sul with a history of high fever for 8 days, temperature peaking $40^{\circ} \mathrm{C}$, especially in the evening and night. The febrile episodes lasted for about 2-3 hours, slightly easing with the use of antipyretics, and were followed by sweating and fatigue during defervescence. Also noticed were prostration, appetite loss and weight loss (approximately $2 \mathrm{~kg}$ in a week), in addition to diarrhea, vomiting and diffuse abdominal pain. Physical examination presented HR (heart rate) $=120 \mathrm{bpm}, \mathrm{RR}$ (respiratory rate $)=36 \mathrm{rpm}$, axillary temperature $=39.5^{\circ} \mathrm{C}$, weight $=$ $27 \mathrm{~kg}$. The patient was pale, anicteric and showing intense prostration. The cardiopulmonary examination showed no noteworthy changes. He presented abdominal distension and relevant visceromegaly, with liver palpable at $6 \mathrm{~cm}$ below the RCM and $10 \mathrm{~cm}$ from the xiphoid process, very painful, and spleen at $4.5 \mathrm{~cm}$ below the left costal margin, painless. Both viscera occupied all mesogaster and hypochondria. The extremities showed no edema, and good peripheral perfusion was seen. The results of blood count and biochemical tests were: hemoglobin concentration $=9.3 \mathrm{~g} / \mathrm{dL}$, hematocrit $=28 \%$, leukocytes $=2900 \mathrm{~mm}^{3}$ (band neutrophils, $4 \%$, segmented neutrophils, $61 \%$, eosinophils, $3 \%$ lymphocytes, $29 \%$ monocytes $3 \%$ ), platelet count $=150,000$, sodium $=136 \mathrm{mmol} / \mathrm{L}$, potassium $=4.4 \mathrm{mmol} / \mathrm{L}$, urea $=49 \mathrm{mg} / \mathrm{dL}$, creatinine $=0.8 \mathrm{mg} / \mathrm{dL} ; \mathrm{AST}=54 \mathrm{U} / \mathrm{L}, \mathrm{ALT}=80 \mathrm{U} / \mathrm{L}, \mathrm{TP}=5.9 \mathrm{~g} / \mathrm{dL}, \mathrm{Alb}=2.5$ $\mathrm{g} / \mathrm{dL}$, Globulins $=3.4 \mathrm{~g} / \mathrm{dL} ; \mathrm{TB}=0,55 \mathrm{mg} / \mathrm{dL}, \mathrm{DB}=0.13 \mathrm{mg} / \mathrm{dL}, \mathrm{IB}$ $=0.42 \mathrm{mg} / \mathrm{dL}$. The occurrence of fever, pallor, hepatosplenomegaly, anemia and leukopenia has initially led to the diagnosis of visceral leishmaniasis (VL), since this disease is endemic in the state of Mato Grosso do Sul. A bone marrow aspirate was collected and examined by an experienced microscopist who discarded Leishmania amastigotes and detected the presence of numerous gametocytes and trophozoites of $P$. falciparum (Figure 1a, b). Blood smears collected on admission were retrospectively analyzed by the same professional, who identified trophozoites and gametocytes of Plasmodium falciparum, initially gone unnoticed (Figure 2a, b).

*Corresponding author: Yvone Maia Brustoloni, Federal University of Mato Grosso do Sul, Campo Grande, Mato Grosso do Sul, Brazil; Tel: +55 673383 4063, E-mail: brustoloni@uol.com.br

Received June 16, 2015; Accepted July 13, 2015; Published July 20, 2015

Citation: Brustoloni YM, Baldissera PM, Brustoloni FM, Dorval MEC (2015) Difficulties in Diagnosis of Malaria in Non-Endemic Areas: A Case Report of a Child in Brazil. Malar Cont Elimination 4: 132. doi: 10.4172/2470-6965.1000132

Copyright: (c) 2015 Brustoloni YM, et al. This is an open-access article distributed under the terms of the Creative Commons Attribution License, which permits unrestricted use, distribution, and reproduction in any medium, provided the original author and source are credited. 


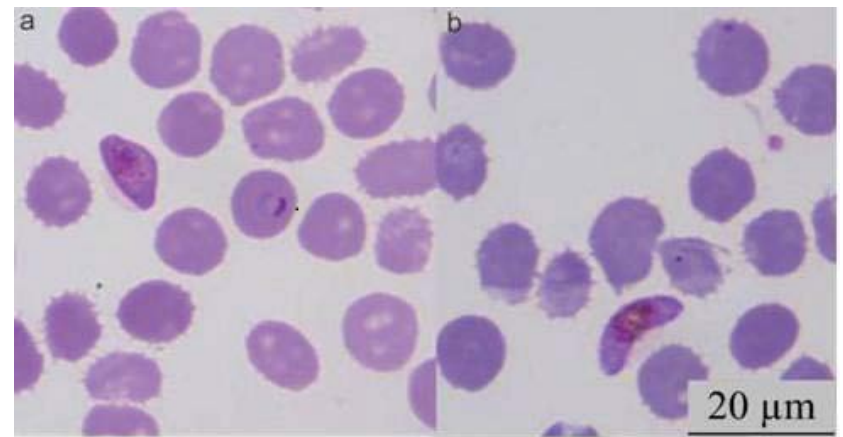

Figure 1: $P$. falciparum gametocyte and trophozoite (A) and gametocyte (B) in bone marrow aspirates.

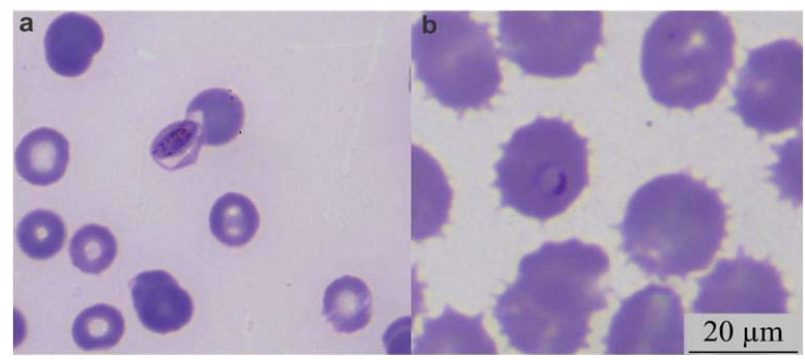

Figure 2: $P$. falciparum gametocyte $(\mathrm{A})$ and trophozoite $(\mathrm{B})$ in peripheral blood smears.

Medical history was reviewed and the child's father reported those 20 days before the onset of symptoms, he had been to the neighboring state of Mato Grosso, where cases of malaria had been diagnosed in other family members. The patient began treatment regimen for P. falciparum, including quinine (500 $\mathrm{mg}$ days 1,2 and 3 ), doxycycline (100 $\mathrm{mg}$ days 1 , $2,3,4$ and 5 ) and primaquine phosphate (15 mg day 6$)$. He also received supportive measures such as intravenous hydration, blood transfusion (when necessary), albumin, and nutritional support. There was a rapid alleviation of symptoms and the patient remained non-febrile from Day 3. One week after the onset of treatment gametocytes of $P$. falciparum were yet seen in the peripheral blood, which disappeared in the course of the second week. The child was discharged in good condition, with recommendation to carry out thick blood smear on a weekly basis for at least 45 days.

\section{Discussion}

In Brazil, about $99.8 \%$ of the malaria cases occur in the states belonging to the "Legal Amazon" [3], the area in the country where malaria is endemic. "Legal Amazon" is a political division comprising nine states in Brazil, all of which belong to the Amazon Basin and therefore encompass the Amazon forest. Mato Grosso do Sul, where the patient at issue resides, does not belong to this region, but the child had recently visited the state of Mato Grosso, which is part of that area. People at the highest risk of acquiring the disease in regions where malaria is non-endemic are those who travel to the areas of transmission [4]. It is in non-endemic regions that malaria is more lethal, because when highly vulnerable people travel to areas of transmission and return infected frequently are exposed to late or incorrect diagnosis. Outside of endemic areas, generally healthcare professionals are not familiar with the disease [3], often failing to include it among the potential diagnostic possibilities or to order laboratory tests that could prove it.
In addition, laboratorians may lack experience with malaria and fail to detect parasites when examining blood smears under the microscope. Both situations have occurred in this case.

Brazil is a country where visceral leishmaniasis is an endemic disease and this explains why the initial diagnosis was addressed to this pathology. Signs and symptoms of malaria often are nonspecific [5], including fever, headache, chills, increased sweating, back pain, myalgia, diarrhea, nausea, vomiting, cough and hepatosplenomegaly. Many diseases and conditions share nonspecific clinical symptoms among them visceral leishmaniasis, hepatitis, tuberculosis, brucellosis, typhoid fever, leukemia and others, requiring differential diagnosis. The child had traveled to a malaria area a few days before the onset of symptoms, but this fact went unnoticed at first. It was not until the parasite in bone marrow aspirate was detected that the issue was raised. Professionals not accustomed to suspect of malaria, when facing a case of fever of undetermined etiology frequently do not interrogate if the patient had recently been in an endemic area of the disease. This report, when obtained by the doctor, leads to the assumption of correct diagnosis of malaria, preventing serious complications and death. Health-care providers should ask all febrile patients for a travel history [5].

Various methods have now been employed for malaria diagnosis [6]. PCR techniques are highly sensitive for the detection of parasite nucleic acids [7-9], are particularly valuable for species confirmation and should be used in all instances when species cannot be determined by microscopy or to evaluate for mixed infections [5] but unfortunately are not always available everywhere. Immunochromatographic tests most often using a dipstick or a cassette format contain monoclonal antibodies against parasite specific antigens and provide results in 5-20 minutes $[10,11]$. Even after the progress of diagnostic techniques, the visualization of parasites in peripheral blood is considered the gold standard for diagnosing malaria. Thick and thin blood films must be prepared. The thick blood smear is the official method employed in Brazil for malaria diagnosis [3]. Giemsa-stained thick blood film include many advantages: is simple, effective, of low cost and implementation; allows the calculation of the parasite density in relation to the microscopic fields examined $[10,12]$; has the potential of differentiating parasite species by analyzing its morphology and presence of different developmental stages; permits to monitor response to therapy; makes possible the storing of the slides for an indefinite period, making feasible the future control of the test quality. Thin smear of peripheral blood presents low sensitivity because a small amount of blood is examined [5], but allows the differentiation of species with more ease and security.

The diagnostic efficacy of thick blood film depends on well-trained technicians with experience in reading the slides. Parasites had already been present in the smears of peripheral blood collected on admission, but had not been noticed. Microscopy technicians frequently have difficulties in recognizing malaria etiologic agent of disease outside the endemic areas. Errors in parasitological diagnosis like false negative and positive are reported $[13,14,10]$. False negative results may lead to the absence of treatment and thus to the remaining of the source of infection for the vector.

Malaria diagnosis in the case here reported was firstly made in bone marrow aspirate instead of in blood. Siddig et al. [15] reported patients with Plasmodium gametocytes identified in bone marrow and/or splenic aspirates of patients with visceral leishmaniasis in Sudan. This shows that skilled technicians are able to recognize malaria parasites when they are trained to. Training microscopy technicians is one of the key measures for controlling malaria. Besides professional training 
and competence, deficiency in microscopy skills has been admitted as a result of multiple factors, including technique in preparing the slides, conditions of the microscope and quality of laboratory supplies. However, even with similar training and equipment, the difference in individual skills between the microscopy technicians is significant and even in developed countries technicians skilled in diagnosing malaria are scarce [10]. The thick blood smear permits the differentiation of Plasmodium species and the identification of the developmental stage of the circulating parasite. A proficient microscopy technician should also be able to properly recognize the species of Plasmodium. The identification is critical for the therapy since treatment should be determined on the basis of the infecting Plasmodium species. Malaria is often a debilitating illness, and when caused by $P$. falciparum a delay in treatment may have fatal consequences, especially for in infants and young children [12]. In Brazil, P.vivax malaria is worrying too, with severe cases emerging and causing an increased number and rate of hospital admissions [16].

\section{Conclusion}

The case reported here illustrates that clinicians need to be aware of considering malaria in the differential diagnosis of pathologies presenting fever and hepatosplenomegaly, asking all patients for a travel history to a malarious area. Technicians must be trained to recognize the etiologic agent of the disease in both endemic and non-endemic areas. Early diagnosis guarantees the adoption of proper and timely therapeutic regimen, representing an effective way of reducing the severity and lethality of malaria.

\section{References}

1. WHO (2011) World Malaria Report.

2. Braz RM, Guimarães RF, Carvalho Júnior OA, Tauil PL (2014) Spatial dependence of malaria epidemics in municipalities of the Amazonian Ecosystem. Rev Bras Epidemiol 17: 615-628.
3. Oliveira-Ferreira J, Lacerda MV, Brasil P, Ladislau JL, Tauil PL, et al. (2010) Malaria in Brazil: an overview. Malar J 9: 115.

\section{WHO (2015) Malaria.}

5. Mali S, Kachur SP, Arguin PM; Division of Parasitic Diseases and Malaria Center for Global Health; Centers for Disease Control and Prevention (CDC) (2012) Malaria surveillance--United States, 2010. MMWR Surveill Summ 61: $1-17$.

6. Lee SH, Kara UA, Koay E, Lee MA, Lam S, et al. (2002) New strategies for the diagnosis and screening of malaria. Int J Hematol 76 Suppl 1: 291-293.

7. Johnston SP, Pieniazek NJ, Xayavong MV, Slemenda SB, Wilkins PP, et al. (2006) PCR as a confirmatory technique for laboratory diagnosis of malaria. J Clin Microbiol 44: 1087-1089.

8. Erdman LK, Kain KC (2008) Molecular diagnostic and surveillance tools for global malaria control. Travel Med Infect Dis 6: 82-99.

9. Lima GF, Levi JE, Geraldi MP, Sanchez MC, Segurado AA, et al. (2011) Malaria diagnosis from pooled blood samples: comparative analysis of real-time PCR nested PCR and immunoassay as a platform for the molecular and serological diagnosis of malaria on a large-scale. Mem Inst Oswaldo Cruz 106: 691-700.

10. Wongsrichanalai C, Barcus MJ, Muth S, Sutamihardja A, Wernsdorfer WH (2007) A review of malaria diagnostic tools: microscopy and rapid diagnostic test (RDT). Am J Trop Med Hyg 77: 119-127.

11. Bisoffi Z, Gobbi F, Buonfrate D, Van den Ende J (2012) Diagnosis of Malaria Infection with or without Disease. Mediterr J Hematol Infect Dis 4: e2012036.

12. WHO (2010) Guidelines For The Treatment of Malaria. (second edition), World Health Organization, Switzerland.

13. Kachur SP, Nicolas E, Jean-François V, Benitez A, Bloland PB, et al. (1998) Prevalence of malaria parasitemia and accuracy of microscopic diagnosis in Haiti, October 1995. Rev Panam Salud Publica 3: 35-39.

14. Thwing J, Skarbinski J, Newman RD, Barber AM, Mali S, et al. (2007) Malaria surveillance - United States, 2005. MMWR Surveill Summ 56: 23-40.

15. Siddig M, Ghalib H, Shillington DC, Petersen EA, Khidir S (1990) Viscera leishmaniasis in Sudan. Clinical features. Trop Geogr Med 42: 107-112.

16. Santos-Ciminera PD, Roberts DR, Alecrim Md, Costa MR, Quinnan GV Jr (2007) Malaria diagnosis and hospitalization trends, Brazil. Emerg Infect Dis 13: $1597-1600$. 